\title{
INFLUÊNCIA DAS CARACTERÍSTICAS OBSTÉTRICAS E MATERNAS NA PREVALÊNCIA DO ALEITAMENTO MATERNO
}

\author{
João Duarte \\ Instituto Politécnico de Viseu \\ Escola Superior de Saúde \\ Paula Nelas \\ Instituto Politécnico de Viseu \\ Escola Superior de Saúde \\ pnelas@gmail.com \\ Emília Coutinho \\ Instituto Politécnico de Viseu \\ Escola Superior de Saúde \\ Cláudia Chaves \\ Instituto Politécnico de Viseu \\ Escola Superior de Saúde \\ Odete Amaral \\ Instituto Politécnico de Viseu \\ Escola Superior de Saúde \\ Rui Dionísio \\ Instituto Politécnico de Viseu \\ Escola Superior de Saúde
}

Fecha de Recepción: 26 Marzo 2019

Fecha de Admisión: 30 Abril 2019

\section{RESUMO}

Enquadramento: Apesar de conhecidas as vantagens do aleitamento materno, a sua manutenção ainda está longe da recomendada, sofrendo influência de fatores psicológicos, biológicos, sociais e culturais. Objetivos: Determinar se as características sociodemográficas e obstétricas influenciam a prevalência do aleitamento materno. Métodos: Estudo quantitativo, transversal, descritivo e correlacional, com uma amostra de 1102 mulheres de Portugal Continental, com idade média de 32 anos $( \pm 5.87)$. 0 protocolo de colheita de dados é 0 questionário que permite a caracterização sociodemográfica e da gravidez. Resultados: A idade materna surge como uma variável determinante na prevalência aos 6 e 24 meses. 0 estado civil apresenta diferenças significativas nas mulheres que amamentam aos 24 meses e têm companheiro. A escolaridade demonstrou ser uma variável determinante na amamentação aos 6 e 24 meses. Relativamente à vigilância da gravidez e 


\section{INFLUÊNCIA DAS CARACTERÍSTICAS OBSTÉTRICAS E MATERNAS NA PREVALÊNCIA DO ALEITAMENTO MATERNO}

ao número de consultas realizadas, verificam-se diferenças estatisticamente significativas na prevalência da amamentação aos seis meses. Assim como o fato de o parto ser eutócico. Por outo lado, não se observaram diferenças significativas na frequência de aulas de preparação para o parto e a prevalência da amamentação aos 6 e 24 meses. Conclusões: Como existe uma multiplicidade de fatores que influência a motivação, a prática e manutenção do aleitamento materno, recomendamos programas que promovam a manutenção. Deve ainda existir apoio dos profissionais de saúde para ajudar a superar as dificuldades encontradas, prevenindo o abandono precoce do aleitamento materno.

Palavras-chave: prevalência; aleitamento materno; gravidez

\section{ABSTRACT}

Influence of obstetric and maternal characteristics on the prevalence of breastfeeding. Background: Although the advantages of breastfeeding are well known, their maintenance is still far from recommended, influenced by psychological, biological, social and cultural factors. Objectives: To determine if sociodemographic and obstetric characteristics influence the prevalence of breastfeeding. Methods: A quantitative, cross - sectional, descriptive and correlational study with a sample of 1102 women from mainland Portugal, with a mean age of 32 years $( \pm 5.87)$. The data collection protocol is the questionnaire that allows sociodemographic and pregnancy characterization. Results: Maternal age appears as a determinant variable in prevalence at 6 and 24 months. Marital status shows significant differences in women who breastfeed at 24 months and have a partner. Schooling has been shown to be a determinant variable in breastfeeding at 6 and 24 months. Regarding pregnancy monitoring and the number of consultations performed, there are statistically significant differences in the prevalence of breastfeeding at six months. As well as the fact that the delivery is eutocic. On the other hand, there were no significant differences in the frequency of prep classes and the prevalence of breastfeeding at 6 and 24 months. Conclusions: Because there is a multiplicity of factors that influence the motivation, practice and maintenance of breastfeeding, we recommend programs that promote maintenance. There should also be support from health professionals to help overcome the difficulties encountered, preventing the early cessation of breastfeeding.

Keywords: prevalence; breastfeeding; pregnancy

\section{INTRODUÇÃO}

A amamentação é 0 ato de extração de leite materno diretamente da mama e sempre pelo bebé (Marques \& Ramalho, 2015). A sua necessidade fisiológica pode perdurar até aos seus primeiros três anos de vida, não havendo consenso no que se refere ao tempo máximo de lactação (Carneiro, Barbieri, Moro, Freitas, Colomé \& Backes, 2014). Todavia preconiza-se que 0 aleitamento materno seja exclusivo até aos seis meses e complementado até aos dois anos de idade ou mais, conforme as recomendações da Organização Mundial de Saúde relativas à amamentação.

0 leite materno é um alimento vivo, único e completo constituindo-se no melhor alimento para recém-nascidos e lactentes, exceto em raras exceções. Apesar do reconhecimento mundial da superioridade do leite materno em relação ao leite artificial, sob o ponto de vista nutricional, imunológico e psicossocial, a prevalência do aleitamento materno tem sido flutuante ao longo dos anos.

Com a finalidade de proteção do aleitamento materno das estratégias publicitárias não éticas, a OMS em 1981 recomenda aos países a adoção do Código Internacional de Marketing dos Substitutos do Leite Materno (Direção-Geral da Saúde, 2008). Em 1990, a mesma organização aliada à UNICEF, com a Declaração Innocenti, direcionam esforços para a instituição de políticas de incentivo à amamentação, surgindo a publicação do texto "Proteção, promoção e apoio ao aleita- 
mento materno: o papel dos serviços de saúde" que expõe os "Dez passos para o aleitamento materno". Em 1992, a OMS e a UNICEF, na sequência da Cimeira Mundial para a Infância, salientando a importância dos primeiros dias de vida do recém-nascido no estabelecimento da amamentação, criaram os Hospitais Amigos do Bebés (HAM), cujos objetivos específicos são a promoção, proteção e apoio ao aleitamento materno. Os membros da OMS, em 2002, na 55a Assembleia Mundial de Saúde, adotaram a Estratégia Global para a Alimentação de Lactentes e Crianças na Primeira Infância.

Face a todos os argumentos relacionados às vantagens do aleitamento materno, sendo importante para a criança, para a mãe, para a família e para a sociedade em geral, seria expectável que a sua prática estivesse em pleno desenvolvimento. Contudo, a realidade no mundo é da persistência do desmame precoce, ou seja, a suspensão do aleitamento materno aos 4 meses (Caminha, Serva, Arruda \& Filho, 2010). No contexto internacional, a prevalência e duração do aleitamento materno são menores em países desenvolvidos e ainda assim nos países em desenvolvimento a prevalência do aleitamento materno é de $37 \%$ com grandes disparidades entre os mais pobres e os mais ricos (Oliveira, Boccolini, Faertein \& Verly-Jr, 2016). Em alguns países da União Europeia as taxas de iniciação ao aleitamento materno são muito baixas e mesmo onde são altas, como nos países escandinavos, verifica-se um elevado abandono nos primeiros seis meses (Lanzaro, Santos, Guerra, Hespanhol \& Esteves, 2015).

Os estudos indicam uma incidência elevada de amamentação à nascença e uma baixa prevalência a partir do mês, mês e meio de vida. Indicam mesmo uma elevada incidência de amamentação na alta hospitalar $(98,5 \%)$ e em exclusivo $(65,2 \%)$, diminuindo consideravelmente no quarto mês $(26,9 \%)$ de aleitamento exclusivo e $(34,0 \%)$ de amamentação predominante (Sardo, 2016), aos seis as taxas de exclusividade variam entre 17 a $34 \%$, longe do objetivo de 50\% proposto pela OMS para 2010 (Lanzaro et al., 2015).

Em Portugal, o Ministério da Saúde no seu Plano Nacional de Saúde 2012-2016, recomenda 0 incentivo desta prática e assume-a como um critério de qualidade dos cuidados de saúde perinatais, ou seja, o mesmo é olhado como o primeiro estilo de vida saudável na vida de uma criança (Direção Geral de Saúde, 2015).

Face ao exposto, questionamo-nos sobre a prevalência do aleitamento materno em função das variáveis sociodemográficas e contextuais à gravidez.

\section{AMOSTRA}

A amostra é constituída por 1102 mulheres de Portugal Continental, sendo o critério de inclusão mulheres que foram mães há pelo menos dois anos. Na seleção da amostra, optou-se por uma amostragem do tipo não probabilista por conveniência, constituída pelas mães que voluntariamente manifestaram interesse em participar no estudo. Foi critério de exclusão as mães que pela sua condição de saúde ou do seu filho foram impedidas de amamentar.

\section{INSTRUMENTO DE COLHEITA DE DADOS}

0 instrumento de colheita de dados foi o questionário constituído por 3 partes: I ParteCaracterização sociodemográfica; II Parte- Caracterização da gravidez; III Parte - Caracterização do aleitamento materno.

A caracterização sociodemográfica permite traçar um perfil sociodemográfico (idade, estado civil, nacionalidade, escolaridade, profissão, situação profissional, remuneração e agregado familiar). A parte relativa às características da gravidez permite apurar a duração da gravidez, caracterização das consultas de vigilância pré natal, frequência de curso de preparação para o parto e parentalidade, existência de conhecimentos sobre aleitamento materno e quem é responsável pela infor- 


\section{INFLUÊNCIA DAS CARACTERÍSTICAS OBSTÉTRICAS E MATERNAS NA PREVALÊNCIA DO ALEITAMENTO MATERNO}

mação, classificação da informação transmitida durante a gravidez, existência de problemas na gravidez, tipo de gravidez, tipo de parto, duração do internamento após o nascimento, número de filhos, número de gravidezes e local de nascimento).

\section{PROCEDIMENTOS ÉTICOS E FORMAIS}

Para esta investigação obteve-se a autorização da Comissão Nacional da Proteção de Dados e das Comissões de Ética da ARS Norte e Centro para a aplicação do instrumento de recolha de dados. Foi ainda garantida a confidencialidade dos dados obtidos.

Este estudo integra um projeto submetido ao Centro de Estudos em Educação, Tecnologias e Saúde - Instituto Politécnico de Viseu; PROJ/CI\&DETS/2016/0017, com o título: Prevalência da amamentação, motivação, dificuldades e a ajuda dos enfermeiros. Projeto financiado pelo CI\&DETS. Foram ainda assegurados os procedimentos éticos e legais. Os dados foram tratados com recurso ao SPSS versão 23.0 para Windows.

\section{CARACTERIZAÇ̃̃O SOCIODEMOGRÁFICA}

A amostra apresenta uma idade mínima de 18 anos e uma máxima de 46 anos, ao que corresponde uma média de idade de 32 anos, com um desvio padrão de 5.87 anos. A maioria (57.1\%) é da região centro do país. A quase totalidade da amostra (97.6\%) é de nacionalidade portuguesa e possui companheiro (93.1). Na amostra, $50.5 \%$ têm escolaridade igual ou superior ao $12^{\circ}$ ano e $35.6 \%$ exerce profissões técnicas, salientando-se que as profissões indiferenciadas apresentam uma representatividade de $29.2 \%$. Encontra-se empregada $83.9 \%$ da amostra. A maioria $(51.5 \%)$ aufere entre 500 a 999 euros por mês. Existe um predomínio de participantes residentes no meio urbano (64.7\%). Quanto à composição do agregado familiar, verifica-se que $45.1 \%$ possui um agregado constituído por três pessoas.

\section{RESULTADOS}

\section{Caracterização da gravidez}

A maioria (87.5\%) teve uma gravidez com duração igual ou superior a 37 semanas e $84.0 \%$ vigiou adequadamente a gravidez. Salienta-se que $57.7 \%$ não frequentou um curso de preparação para o parto e parentalidade. A maioria (85.7\%) recebeu informação sobre aleitamento materno, sendo que $89.4 \%$ diz que a informação foi facultada pelos enfermeiros. Os resultados indicam que $47.2 \%$ das participantes considera que a qualidade da informação transmitida durante a gravidez foi moderada, secundadas pelas que a classificam como fraca (26.6\%). A maioria (83.3\%) não teve problemas durante a gravidez. A quase totalidade da amostra (98.0\%) refere gravidez de feto único. Na amostra, 59.3\% teve parto eutócico. Mais de metade (81.9\%) teve um internamento após o parto igual ou superior a três dias. Constata-se que 51.5\% têm apenas um filho.

\section{Caracterização do aleitamento materno}

Na amostra, $54.9 \%$ das participantes refere que o bebé apenas foi alimentado com leite materno, $8.5 \%$ com leite artificial e $36.6 \%$ expressa que o bebé fez aleitamento misto. Apenas $76.2 \%$ amamentou na primeira hora de vida. Houve contacto pele a pele após o nascimento em $79.7 \%$ das díades. Das participantes que fizeram contacto pele a pele, $72.3 \%$ informa que este durou até 30 minutos e $14.0 \%$ que o mesmo durou até 60 minutos. Verifica-se que $76.8 \%$ das participantes admitem ter amamentado outros filhos. No estudo, $88.8 \%$ das participantes refere não ter sido influenciada por ninguém para amamentar e $6.0 \%$ atribui essa influência ao enfermeiro. 


\section{Caracterização da prevalência do aleitamento materno}

Verifica-se que as maiores prevalências se encontram até aos seis meses de idade do bebé, salientando-se $95 \%$ de bebés amamentados ao mês de vida, $83.7 \%$ aos três meses e $69.1 \%$ aos seis meses. Aos nove meses a prevalência da amamentação desce para $53.7 \%$, sendo aos doze meses de $40.6 \%$. Aos 15 meses a prevalência é de $29.3 \%$ e de $21.7 \%$ aos 18 meses. Observam-se prevalências mais baixas aos 21 e 24 meses (14.8\% e 13.2\%, respetivamente). De salientar que $5.0 \%$ das participantes refere não ter amamentado. Conclui-se que $69.1 \%$ das inquiridas amamenta aos seis meses de vida do seu bebé, porém apenas $13.2 \%$ o faz aos 24 meses. 0 que determina que $86.8 \%$ dos bebés aos 24 meses já não são amamentados.

\section{RESULTADOS}

Verifica-se que as participantes que amamentam aos seis meses, $65.8 \%$ têm idade inferior a 35 anos e $75.3 \%$ têm 35 ou mais anos. Relativamente, à prevalência da amamentação aos 24 meses, observa-se também a existência de diferença estatisticamente significativa entre as mulheres com 35 ou mais anos e que amamentam aos 24 meses. Ao analisarmos a prevalência da amamentação aos seis e 24 meses em função da nacionalidade não se observaram diferenças estatisticamente significativas.

Quando se procede à avaliação das prevalências em função do estado civil, constatamos que aos seis meses existe uma distribuição homogénea de $69.4 \%$ e $65.8 \%$, respetivamente, nas participantes com e sem companheiro. Por outro lado, apura-se uma diferença estatisticamente significativa no grupo que amamenta aos 24 meses e que têm companheiro e nas que não amamentam e não têm companheiro.

Relativamente à prevalência da amamentação em função das habilitações literárias observa-se que aos seis meses a maior percentagem de amamentação ocorre nas participantes com maiores habilitações literárias $(74.6 \%)$, com diferença estatisticamente significativa, em oposição ao grupo com menores habilitações literárias, que já não amamenta aos seis meses (36.2\%). 0 mesmo se verifica aos 24 meses, onde são as participantes com maiores habilitações académicas que mais amamentam.

Relacionando a prevalência com o local de residência, observa-se que $68.6 \%$ das mulheres que amamentam aos seis meses reside em meio urbano e $70.2 \%$ em meio rural, não se verificando, no entanto, diferença estaticamente significativa. No que respeita à amamentação aos 24 meses de idade a distribuição é ainda mais homogénea, com 13.2\% a residir em meio urbano e $13.1 \%$ em meio rural.

Ao analisar-se a amamentação em função da vigilância da gravidez pode concluir-se que existem diferenças significativas $(p=0.000)$ e que estas se encontram entre as participantes que amamentam aos seis meses e tiveram uma gravidez vigiada.

Existe relevância estatística quando se compara a prevalência da amamentação aos 6 e 24 meses com o número de consultas ao longo da gravidez, com os valores residuais a indicarem que as participantes que tiveram cinco ou mais consultas amamentam aos 6 meses, quando comparadas com as que tiveram menos de 5 consultas. Por outro lado, verifica-se não haver diferenças estatisticamente significativas em relação à amamentação aos 24 meses e 0 número de consultas de vigilância pré natal.

Também, $75.3 \%$ das participantes que amamenta aos seis meses assistiu a aulas de preparação para o parto e parentalidade, onde $71.0 \%$ amamenta aos seis meses ainda que raramente tenha frequentado as aulas. No entanto, não existem diferenças significativas entre as mães que amamentam aos seis meses e a frequência das aulas de preparação para o parto $(p=0.448)$. Relativamente à pre- 


\section{INFLUÊNCIA DAS CARACTERÍSTICAS OBSTÉTRICAS E MATERNAS NA PREVALÊNCIA DO ALEITAMENTO MATERNO}

valência da amamentação aos 24 meses, 85.4\% assistiram a aulas de preparação para o parto. Por outro lado, 14.5\% amamentam aos 24 meses sem frequência de aulas de preparação para 0 parto, não se observando diferenças estatisticamente significativas $(p=0.980)$.

Pesquisou-se se existia relação entre a prevalência da amamentação e o tipo de parto, observando-se diferença estatisticamente significativa no grupo das mulheres que experienciaram um parto eutócico e que amamentam aos seis meses $(p=0.000)$. Em oposição a prevalência da amamentação aos 24 meses não está relacionada com o tipo de parto, sendo de 14.5\% nas participantes que tiveram parto eutócico e de $11.1 \%$ nas que experimentaram um parto distócico.

A maioria das mães (74.3\%) sentiu-se preparada para amamentar, solicitando ajuda perante dificuldades $(74.7 \%)$, tendo $80.3 \%$ recorrido à ajuda do enfermeiro.

\section{DISCUSSÃO DOS RESULTADOS}

Em relação à prevalência do aleitamento materno conclui-se que 5.0\% da amostra refere não ter amamentado. Verifica-se que as maiores prevalências se encontram até aos seis meses de idade do bebé, salientando-se $95 \%$ ao mês de vida, $83.7 \%$ aos três meses e $69.1 \%$ aos seis meses. Tendência também revelada no estudo de Ferreira, Oliveira, Bernardo, Almeida, Aquino, Pinheiro (2018). onde a prevalência do aleitamento materno exclusivo ao longo dos seis meses de vida da criança foi caracterizada por uma linha descendente, sendo de $89,03 \%$ no primeiro mês de vida e de $30,03 \%$ aos seis meses. Contudo, as prevalências obtidas mostram-se superiores às encontradas em outros estudos. A Direção Geral de Saúde (2014) apresenta 88.1\% de amamentação à 5a semana de vida, $76.7 \%$ aos três meses, $63.6 \%$ aos quatro meses e $53.9 \%$ aos seis meses. Por outro lado, Lanzaro, et al. (2015), apresenta $50.0 \%$ de prevalência do aleitamento materno aos seis meses. Oliveira (2016) obteve uma taxa de aleitamento aos três meses de 56,7\% e aos 6 meses de 40\%. Sardo (2016) indica uma elevada incidência de amamentação na alta hospitalar (98,5\%) e em exclusivo $(65,2 \%)$, diminuindo consideravelmente no quarto mês $(26,9 \%)$ de aleitamento exclusivo e $(34,0 \%)$ de amamentação predominante.

Efetivamente aos nove meses a prevalência da amamentação desce para $53.7 \%$, sendo aos doze meses de $40.6 \%$. Aos 15 meses a prevalência é de $29.3 \%$ e de $21.7 \%$ aos 18 meses. Observam-se prevalências mais baixas aos 21 e 24 meses (14.8\% e 13.2\%, respetivamente).

Verificou-se que as mulheres com mais de 35 anos amamentam até mais tarde, sendo assim a idade fator determinante na amamentação. Dados discordantes foram obtidos por Oliveira, Boccolini, Faertein \& Verly-Jr (2016) em que dos fatores associados ao risco de interrupção do aleitamento materno destacaram a idade da mãe, onde mães com idade mais elevada apresentam maior risco para desmame.

Ao analisar-se a prevalência da amamentação aos seis e 24 meses em função da nacionalidade da mãe, verifica-se não haver diferença estatisticamente significativa, sendo a prevalência aos 6 meses de $69.2 \%$ nas portuguesas e $65.4 \%$ estrangeiras. No que respeita à prevalência aos 24 meses, observa-se que as mulheres portuguesas amamentam em maior número do que as estrangeiras (13.4\% para 3.8\%). Grant (2016) revela num estudo realizado no Reino Unido que mães jovens, brancas, provenientes de zonas carenciadas têm menor probabilidade de amamentar.

Quando se procede à avaliação das prevalências em estudo em função do estado civil, constata-se que aos seis meses existe uma distribuição homogénea de $69.4 \%$ e $65.8 \%$ nas mulheres com e sem companheiro, respetivamente. Por outro lado, apura-se uma diferença estatisticamente significativa no grupo das mulheres que amamentam aos 24 meses e que têm companheiro (14.0\%), e nas que não amamentam aos 24 meses e não têm companheiro $(p=0.002)$. Dados corroborados por Alves, Couto de Oliveira e Rito (2018) que verificaram que não viver com o companheiro está 
associado a uma prevalência de aleitamento materno exclusivo 24\% inferior.

Aos seis meses observa-se que a maior percentagem de amamentação $(74.6 \%)$ ocorre em mulheres com maiores habilitações literárias, existindo uma diferença estatisticamente significativa, que se encontra neste grupo e em oposição no grupo das mulheres com menores habilitações literárias e que já não amamentam aos seis meses ( $p=0.000)$.

Também na amamentação aos 24 meses se verifica 0 mesmo, sendo de $10.0 \%$ para as mães com escolaridade inferior ou igual ao $12^{\circ}$ ano e de $16.4 \%$ para as que têm formação académica superior ao $12^{\circ}$ ano. Pelo que as habilitações literárias maternas interferem na prevalência da amamentação. Diferentes estudos apontam neste sentido, Alves, Couto de Oliveira e Rito (2018) revelam uma menor prevalência de aleitamento materno exclusivo em mães com escolaridade inferior ao ensino médio, até 10 anos completos $(p<0.20)$. A maior escolaridade materna parece ser determinante para 0 êxito da prática do aleitamento materno, podendo estar relacionada com uma maior autoconfiança materna e maior capacidade de obter informação para ultrapassar desconfortos e problemas do ato de amamentar.

Observa-se que $68.6 \%$ das mulheres que amamentam aos seis meses reside em meio urbano e $70.2 \%$ em meio rural, não se verificando diferença estaticamente significativa. No que respeita à amamentação aos 24 meses de idade a distribuição é ainda mais homogénea, com 13.2\% das mulheres a residir em meio urbano e $13.1 \%$ em meio rural. Também Lanzaro at al. (2015) não verificou diferenças entre as populações urbanas e rural, com taxas de amamentação aos seis meses de $50,0 \%$.

Ao analisar-se a amamentação com a vigilância da gravidez verificam-se diferenças significativas $(p=0.000)$ e que estas se encontram entre as mulheres que amamentam aos seis meses e tiveram uma gravidez vigiada, o que revela que a vigilância da gravidez está associada à prevalência da amamentação.

Existe relevância estatística quando se compara a prevalência da amamentação aos seis e 24 meses com 0 número de consultas ao longo da gravidez $(p=0.000)$. Também num estudo de Raheem (2014) receber apoio de um profissional de saúde na amamentação, onde se incluem a consultas de gravidez, surge como fator determinante na prevalência da amamentação. Por outro lado, não existem diferenças estatísticas em relação à amamentação aos 24 meses.

Constata-se que $75.3 \%$ das que amamenta aos seis meses assistiu a aulas de preparação para o parto e parentalidade e que $71.0 \%$ amamenta aos seis meses ainda que raramente tenha frequentado as aulas, não existindo diferenças estatísticas significativas. Contrariamente, no estudo de Alves, Couto de Oliveira e Rito (2018) o aleitamento materno exclusivo aos seis meses surge associado ao ter recebido orientação sobre aleitamento materno exclusivo, em ambiente de consulta, grupos educativos ou visita domiciliária. Relativamente à prevalência da amamentação aos 24 meses, $85.4 \%$ das mães que amamentam os seus bebés aos 24 meses assistiram a aulas de preparação para o parto e parentalidade. Por outro lado, também 14.5\% amamentam aos 24 meses sem frequência de aulas, pelo que também não se observam diferenças estatisticamente significativas. Dados corroborados pol Lanzaro et al. (2015) que também não verificou relação entre a duração da amamentação e frequência de aulas de preparação para o parto.

Observa-se uma relação entre a prevalência da amamentação e o tipo de parto, observando-se diferença estatisticamente significativa no grupo das mulheres que experienciaram um parto eutócico e que amamentam aos seis meses $(p=0.000)$. 0 que é corroborado por Mugadza, Zvinavashe, Gumbo, Stray-Pedersen e Haruzivishe (2016), que no seu artigo de revisão de literatura, apresentam um estudo em que o parto vaginal influencia positivamente 0 início precoce da amamentação na primeira hora de vida. Também Lanzaro et al. (2015), numa análise multivariada, verificou que a 


\section{INFLUÊNCIA DAS CARACTERÍSTICAS OBSTÉTRICAS E MATERNAS NA PREVALÊNCIA DO ALEITAMENTO MATERNO}

maior duração do aleitamento materno teve uma relação significativa com parto por via vaginal. Em oposição a prevalência da amamentação aos 24 meses não está relacionada com 0 tipo de parto vivenciado.

Relacionando a prevalências da amamentação aos seis e 24 meses com o número de filhos, não se observa relevância estatisticamente significativa. Verifica-se uma distribuição semelhante em ambos os grupos, sendo de $66.7 \%$ e $71.8 \%$ para as mães com um ou dois ou mais filhos respetivamente. De igual modo, aos 24 meses amamentam 12.3\% e 13.2\% das mulheres com um ou dois e mais filhos. Muito embora, não se tenha verificado relação entre o número de filhos e a prevalência da amamentação, a literatura refere que a existência de experiências anteriores de amamentação bem-sucedida podem não garantir, mas possivelmente interferem positivamente na decisão e na prática de aleitamento materno com filhos posteriores. No presente estudo $76.8 \%$ das mães refere ter amamentado outros filhos, podendo este facto estar na origem das prevalências elevadas de amamentação apuradas.

\section{CONCLUSÃO}

A pertinência deste estudo prende-se com o facto de o aleitamento materno ser encarado como uma prioridade de saúde. A sua prática é considerada pela OMS/UNICEF uma recomendação mundial de saúde pública, sendo reconhecido o importante papel dos enfermeiros na sua promoção, proteção e apoio, mantendo o foco nas dificuldades sentidas pela mulher que amamenta, no sentido de as ajudar a ultrapassar as dificuldades que podem comprometer a duração da amamentação. É importante ainda ter conhecimento dos contextos de amamentação, para rastrear a influência de múltiplas variáveis podem influenciar a prevalência da amamentação.

Os cuidados e apoio prestados pelos profissionais de saúde durante o período de amamentação permitem 0 acompanhamento das famílias ao longo do ciclo vital, nomeadamente na fase de transição para a parentalidade e refletem a conceção das famílias como unidades a ser cuidadas a fim de construir movimentos que as fortaleçam e possibilitem um viver saudável.

\section{REFERENCIAS BIBLIOGRÁFICAS}

Alves, J. S., Couto de Oliveira, M. I., \& Rito, R. V. V. F. (2018). Orientações sobre a amamentação na atenção básica de saúde e associação com o aleitamento materno exclusivo. Ciências \& Saúde Coletiva, 23(4), 1077-1088. Acedido em http://www.scielo.br/pdf/csc/v23n4/1413-8123-csc-2304-1077.pdf

Caminha, M. F. C., Serva, V. B., Arruda, I. K. G., \& Batista Filho, M. (2010). Aspetos históricos, científicos, socioeconómicos e institucionais do Aleitamento Materno. Revista Brasileira Saúde Materna Infantil, 10(1), 25-37. Acedido em http://www.scielo.br/pdf/rbsmi/v10n1/v10n1a03.pdf

Carneir, L. M. M. C., Barbieri, F., Moro, A. S. S., Freitas, H. M. B., Colomé, J. S., \& Backes, D. S. (2014). Prática do Aleitamento materno por puérperas: Factores de risco para o desmame precoce. Disciplinarum Scientia: Ciências da Saúde, (15)2, 239-248. Acedido em https://periodicos.ufn.edu.br/index.php/disciplinarumS/article/view/1085/1028

Ferreira, H. L. O. C., Oliveira, M. F., Bernardo, E. B. R., Almeida, P. C., Aquino, P. C., \& Pinheiro, A. K. B. (2018). Fatores à adesão ao aleitamento materno exclusivo. Ciências \& Saúde Coletiva, 23(3), 683-690. Acedido em http://www.scielo.br/pdf/csc/v23n3/1413-8123-csc-23-030683.pdf

Grant, A. (2016). I...don t want to see you flashing your bits around: exibitionism, othering andgood motherhood in perceptions of public breastfeeding. Geoforum, 71, 52-61. Acedido em https://www.sciencedirect.com/science/article/pii/S0016718515301627 
Lanzaro, C., Santos, P., Guerra, A., Hespanhol, A. P., \& Esteves, M. J. (2015). Prevalência do aleitamento materno: Comparação entre uma população urbana e uma população rural do norte de Portugal. Acta Pediátrica Portuguesa, 46(2), 101-108. Acedido em https://pjp.spp.pt//article/view/3612/5072

Mugadza, G., Zvinavashe, M., Gumbo, F. Z., Stray-Pederso, B., \& Harusivishe, C. (2016). Early breastfeedindinitiation (EBFI). International Journal of Nursing and Midwifery, 8(10), 81-85. Acedido em https://academicjournals.org/journal/IJNM/article-full-text/007B58E62179

Oliveira, M. (2016). Aleitamento materno: Estudo de prevalência e fatores condicionantes nos primeiros seis meses de vida. Pensar Enfermagem, 20(1), 4-15. Acedido em http://pensarenfermagem.esel.pt/files/Artig01_4_15.pdf

Oliveira, M. (2016). Aleitamento materno: Estudo de prevalência e fatores condicionantes nos primeiros seis meses de vida. Pensar Enfermagem, 20(1), 4-15. Acedido em http://pensarenfermagem.esel.pt/files/Artig01_4_15.pdf

Oliveira, S., Boccolini, C. S., Faestein, E., \& Verly- Jr, E. (2017). Duração do aleitamento materno e fatores associados entre 1960 e 2000. Jornal de Pediatria, 93(2), 130-135. Acedido em http://www.scielo.br/pdf/jped/v93n2/pt_0021-7557-jped-93-02-0130.pdf

Portugal, Ministério da Saúde, Direção-Geral da Saúde. (2008). Código Internacional de marketing de substitutos de leite materno (Ed. revista). Lisboa: DGS. Acedido em http://www.saudereprodutiva.dgs.pt/publicacoes/infeccoes-de-transmissao-sexual/a5-codigo-versao-final_26-0509.aspx

Portugal, Ministério da Saúde, Direção-Geral da Saúde. (2014). Registo do aleitamento materno: Relatório Janeiro a dezembro 2013. Lisboa: Divisão de Saúde Sexual, Reprodutiva Infantil e Juvenil. Lisboa: DGS. Acedido em https://www.dgs.pt/documentos-e-publicacoes/iv-relatoriocom-os-dados-do-registo-do-aleitamento-materno-2013.aspx

Portugal, Ministério da Saúde, Direção-Geral de Saúde. (2015). Programa nacional para a vigilância da gravidez de baixo risco. Lisboa: DGS. Acedido em https://www.dgs.pt/em-destaque/programa-nacional-para-a-vigilancia-da-gravidez-de-baixo-risco.aspx

Raheem, R. A. (2014). A cohort study of postnatal depression, infant feeding practices and infant growth in Male', the Republic of Maldives (Tese de doutoramento, Curtin University, Australia). Acedido em https://espace.curtin.edu.au/handle/20.500.11937/1591

Sardo, D. (2016). Intrinsic and extrinsic motivation to breastfeed scale: Adaptation and validation for portuguese population. Procedia-Social and Behavioral Sciences 217, 1133-1141. Acedido em https://www.researchgate.net/publication/294259990_Intrinsic_and_extrinsic_motivation_to_b reastfeed_scale_Adaptation_and_validation_for_Portuguese_population 
